\title{
ANEMIA AND ASSOCIATED FACTORS AMONG PREGNANT WOMEN ATTENDING ANTENATAL CARE CLINIC IN WOLAYITA SODO TOWN, SOUTHERN ETHIOPIA
}

\author{
Lealem Gedefaw ${ }^{1}$, Asrat Ayele ${ }^{1}$, Yaregal Asres ${ }^{1}$, Andualem Mossie ${ }^{2}$
}

ABSTRACT

BACKGROUND: Anemia during pregnancy is a common problem which affects both the mother's and her child's health. The main aim of the study was to determine the prevalence and associated risk factors of anemia among pregnant women.

METHODS: We conducted a facility based cross-sectional study on 363 pregnant women attending antenatal care clinic in Wolayita Soddo Otona Hospital from January to March 2014. Sociodemographic data were collected through questionnaire based interview. Four milliliter of venous blood and five grams of fecal samples were collected from each pregnant woman. Hematological parameters were determined using CELL DYN $1800^{\circledR}$ (Abott, USA) Hematology analyzer. Stool samples were checked for intestinal parasites using both direct wet mount and formol-ether concentration techniques. Data were analyzed using SPSS version 20 software.

RESULTS: Overall, the prevalence of anemia was 39.94\% (95\% CI: 34.7 - 45.2\%), of which the majority (60\%) had moderate anemia. The mean hemoglobin concentration was $11.55 \pm 2.97 \mathrm{~g} / \mathrm{dl}$. Age 15-24 years (AOR: 9.89, 95\%CI:2.68-21.41), family size >5 (AOR:7.74, 95\%CI:4.15-16.47), multigravida (AOR:2.66, 95\%CI:1.1.31-4.53), having low income (AOR:5.81, 95\%CI:2.93-14.11), current clinical illness (AOR: 6.38, 95\%CI:3.13-13.00), intestinal parasitic infection (AOR:2.41, 95\%CI:1.08-5.81), no history of contraceptive usage (AOR:5.02 95\%CI:2.21-11.47), being in third trimesters (AOR:11.37, 95\%CI:4.5624.82), history of excess menstrual bleeding (AOR:9.82, 95\%CI:3.27-21.35) and low body mass index (AOR:9.44, 95\%CI:7.79-22.18) were identified as independent predictors of anemia among pregnant women.

CONCLUSION: Anemia prevalence was found out to be moderate public health importance. Identified risk factors should be considered for prevention and control of anemia among pregnant women.

KEYWORDS: Hemoglobin, Anemia, Pregnant women, Antenatal care clinic

DOI: http://dx.doi.org/10.4314/ejhs.v25i2.8

\section{INTRODUCTION}

Anemia in pregnancy is a major public health problem, especially in developing countries. It affects $41.8 \%$ of pregnant women globally, with the highest prevalence in Africa (57.1\%) which corresponds to 17.2 million (1). Different studies have shown different prevalence of anemia during pregnancy ranging from 16.6-95.0\% (1-12). Anemia in pregnant women has severe consequences on health, social, and economic development $(1,2)$. Anemic pregnant women will be at risk of low physical activity, increased maternal morbidity and mortality, especially those with severe anemia $(1,10)$. In addition, both pregnant women and their neonates encounter negative consequence including fetal anemia, low birth weight (LBW), preterm delivery, intrauterine growth restriction and perinatal Mortality $(4,10-$ $15)$. The cause of anemia in pregnancy is multifactorial. Iron, folate, vitamin $\mathrm{B}_{12}$ and vitamin $\mathrm{A}$

\footnotetext{
${ }^{1}$ Department of Medical Laboratory Science and Pathology, Jimma University, Ethiopia

${ }^{2}$ Department of Biomedical Sciences, Jimma University, Ethiopia

Corresponding Author: Lealem Bimrew, Email: lealem.gedefaw@ju.edu.et
} 
deficiencies as well as intestinal parasitic infections, malaria, chronic illness have all been shown to be the main causes of anemia among pregnant women $(2,3,10,16)$.

In most of the cases, anemia is largely preventable and easily treatable if detected in time. Effective management of anemia includes treatment of the underlying causes, restoration of the hemoglobin concentration to normal levels, and prevention and treatment of complications. Despite this fact, anemia still continues to be a common cause of mortality and morbidity among pregnant women (17), and data on relative contributions of associated factors are limited which makes it difficult to effectively address the problem. Besides, different studies indicate significant variations in prevalence of anemia both within and between countries which indicates a need for local data to help the preventive program. Furthermore, data on prevalence and associated factors of anemia is limited in the study area particularly in pregnant women. Therefore, this study was aimed at determining the prevalence and associated risk factors of anemia among pregnant women.

\section{METHODS}

Study area and period: A facility based crosssectional study was conducted in Wolayita Sodo Otona Hospital, from January 18 to March 17, 2014. Wolayita Sodo Town is located in southern part of Ethiopia at a distance of $390 \mathrm{~km}$ from capital Addis Ababa. According to the 2007 census conducted by the Central Statistics Agency (CSA), the town has a total population of 76,050 , of whom 40,140 are men and 35,910 are women.

Sample size and sampling technique: The sample size was calculated using the general formula for a single population proportion. This was computed by considering $38.2 \%$ prevalence of anemia among pregnant women (9), 5\% margin of error and 95\% confidence interval (CI). Accordingly, the final sample size was 363. All pregnant women were included during the study period consecutively until the required sample size was obtained. Pregnant women who were on treatment of anemia and who had blood transfusion within four months of data collection were excluded.
Data collection: Data were collected by two trained clinical nurses. Socio-demographic and other related data were obtained using structured questionnaire. Four $\mathrm{ml}$ of EDTA (Ethylene diamine tetra acetic acid) anticoagulated venous blood was collected for hematological analysis. Hemoglobin $(\mathrm{Hb})$ concentration, mean cell volume $(\mathrm{MCV})$, mean cell hemoglobin $(\mathrm{MCH})$ and mean cell hemoglobin concentration (MCHC) were measured by CELL DYN $1800^{\circledR}$ (Abott Laboratories Diagnostics Division, USA). Pregnant women having $\mathrm{Hb}$ value below $11 \mathrm{~g} / \mathrm{dl}$ were considered as anemic. Pregnant women who had $\mathrm{Hb}$ value between $10-10.9 \mathrm{~g} / \mathrm{dl}, 7-9.9 \mathrm{~g} / \mathrm{dl}$ and $<7 \mathrm{~g} / \mathrm{dl}$ were considered as mildly, moderately and severely anemic, respectively (17). Microcytosis was defined when MCV value is below 80fl, and hypochromia was defined when MCHC value is below $32 \mathrm{~g} / \mathrm{dl}$.

Five gm of stool samples were collected from each study participant using clean, wide mouthed and leak proof stool cup. Then, stool samples were examined within 10-15 minutes of collection by wet mount preparation. Leftover samples were processed for formol-ether concentration technique.

At the same day of blood and stool sample collection, each pregnant woman was requested to have weight and height measured by data collectors. Anthropometric measurements of height and weight were measured with women in light clothing. Weight measurement was taken using digital scale to the nearest $0.1 \mathrm{~kg}$, and fixed base scale was used for height measurement to the nearest $0.1 \mathrm{~cm}$. Every height and weight measurement was performed two times; the mean values were used for body mass index (BMI) calculation. BMI was computed as weight in kilogram divided by the square of height in meter using Microsoft Excel office 2007.

Data analysis: All data from the laboratory report forms and questionnaire were checked for completeness, edited, and coded. Then, all the data were entered and analyzed using SPSS-V.20 statistical software. Descriptive statistics were used to give a clear picture of background variables like age and to determine the prevalence of anemia. Binary and multiple logistic regressions were done to identify independent predictors of anemia. All explanatory variables that were associated with the 
outcome variable in bivariable analyses at $25 \%$ level of significance were entered into backward multiple logistic regression model, and then variables significant at $\mathrm{P}$-value $<0.05$ were identified as independent predictors for anemia in pregnant women. Finally, the results were presented using tables and graphs.

To assure quality of the data, training was given to the data collectors to minimize technical and observer bias. Standard operating procedures were followed during specimen collection and laboratory procedures. Control reagents were run to check the accuracy and precision of the data generated by the hematology analyzer.

Ethical clearance and consent: Ethical clearance was obtained from Jimma University Ethical Review Committee. Permission was obtained from Otona Hospital medical director office before data collection. Written informed consent was obtained from each pregnant woman prior to enrollment in the study. Individual level medical information obtained from the pregnant women was kept strictly confidential, and the participants were assured that only aggregate data will be reported. Anemic pregnant women and pregnant women with intestinal parasitic infections and malaria were immediately communicated to the attending health professionals at the ANC clinic of the hospital, for treatment and follow up.

\section{RESULTS}

Description of study participants: A total of 363 pregnant women were involved in the study. Most of the pregnant women were within the age group of 25-35 years with mean age of 28.94 years $( \pm 5.39$ year $)$. The family size of study participants ranged from 1 to 8 with an average of 4.81 persons per household. Among all study participants, 25(6.89\%) were illiterate (Table 1). The mean household income of the participants was 968 ranging from 350 to 3, 600 Ethiopian Birr. Peripheral blood film and stool samples were checked for hemoparasites and intestinal parasites. Accordingly, 13(3.58\%) were confirmed malaria positive, and $69(19.01 \%)$ were found infected with intestinal parasites (Table 2). A total of seven species of intestinal parasites were identified among which Ascaris lumbricoides took the highest proportion (Figure 1).

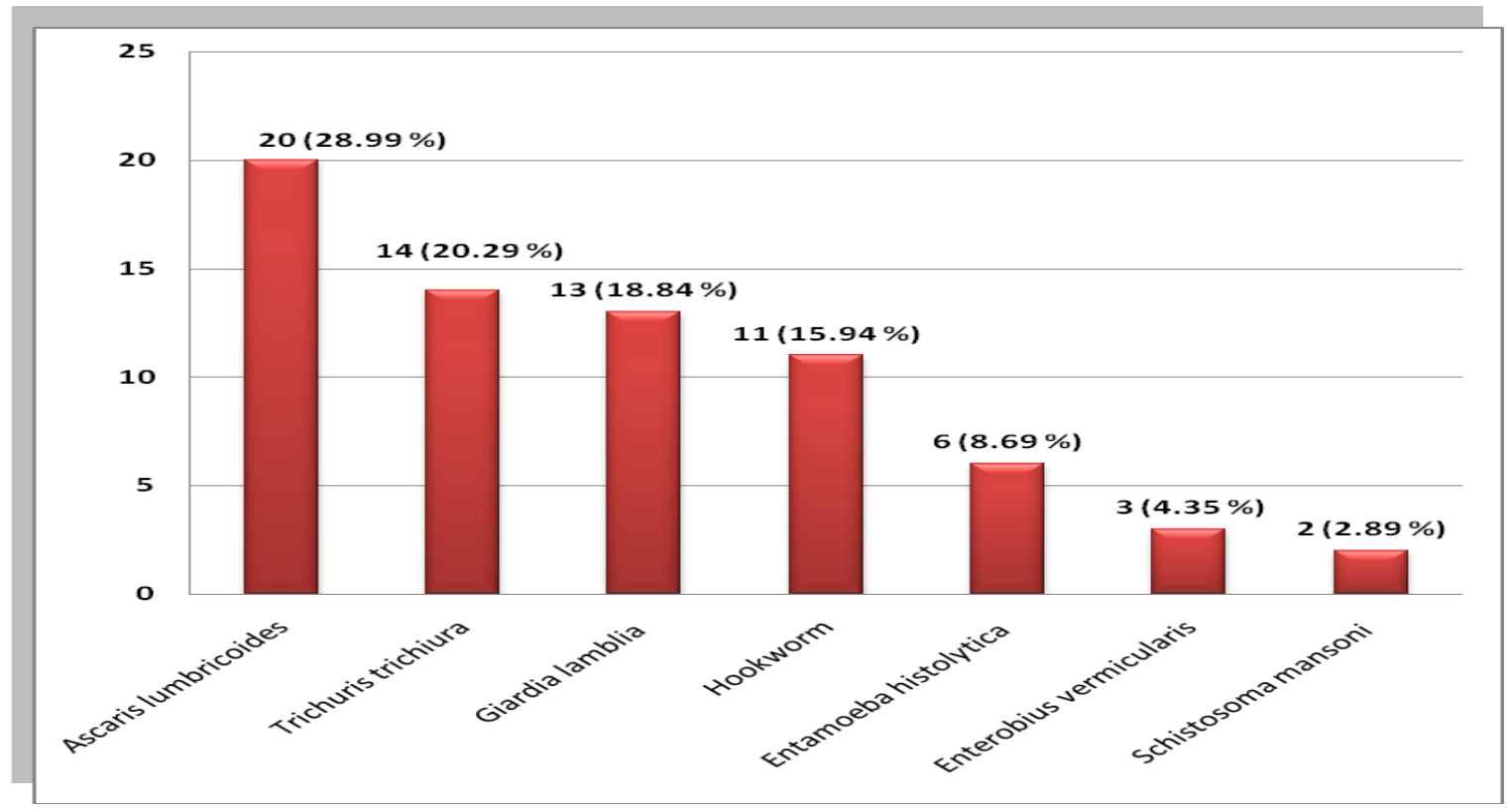

Figure 1: Proportion of different species of intestinal parasite among pregnant women attending ANC clinic in Wolayita Soddo Otona Hospital, Southern Ethiopia: January to March 2014. 
Prevalence and severity of anemia: One hundred and forty five pregnant women, 39.94\% (95\% CI: $34.7 \%$ - 45.2\%), were anemic. The mean $\mathrm{Hb}$ concentration was $11.55 \pm 2.97 \mathrm{~g} / \mathrm{dl}$ with a range of 5.4 to $18.7 \mathrm{~g} / \mathrm{dl}$. Among anemic pregnant women, 44(30.34\%) had mild anemia, $87(60 \%)$ had moderate anemia, and $14(9.66 \%)$ had severe anemia. From a total of anemic pregnant women, $109(75.17 \%), \quad 32(22.07 \%)$ and $4(2.76 \%)$ had normocytic normochromic, microcytic hypochromic and macrocytic anemia, respectively.

Multiple logistic regression analysis was performed to identify independent predictors of anemia among pregnant women. Fourteen explanatory variables that were associated with anemia in bivariable analyses at $25 \%$ level of significance were entered into backward multiple logistic regression model (Tables 1 and 2). In the last step of analysis, four variables were excluded. Accordingly; age 15-24 (AOR: 9.89, 95\% CI:2.68-21.41), family size >5 (AOR:7.74, 95\%CI:4.15-16.47), multigravida (AOR:2.66, 95\%CI:1.1.31-4.53), having low income (AOR:5.81, 95\%CI:2.93-14.11), current clinical illness (AOR: 6.38, 95\%CI:3.13-13.00), intestinal parasitic infection (AOR:2.41, 95\%CI:1.08-5.81), no history of contraceptive usage (AOR:5.02 95\%CI:2.21-11.47), being in third trimesters (AOR:11.37, 95\%CI:4.56-24.82), history of excess menstrual bleeding (AOR:9.82, 95\%CI:3.27-21.35) and low body mass index (AOR:9.44, 95\%CI:7.79-22.18) were identified as independent predictors of anemia among pregnant women (Tables 1 and 2).

Table 1: Association of anemia with socio-demographic and socio-economic factors among pregnant women attending ANC clinic in Wolayita Town, Southern Ethiopia: January to March 2014

\begin{tabular}{llllcc}
\hline \multirow{2}{*}{ Variables } & Categories & \multicolumn{2}{c}{ Anemia } & COR $(95 \%$ C.I) & AOR(95\% C.I) \\
\cline { 3 - 4 } & & Yes (\%) & No $(\%)$ & & \\
\hline Age & $15-24$ & $36(44.44)$ & $45(55.56)$ & $1.84(0.89,3.77)$ & $9.89(2.68,21.41)$ \\
& $25-35$ & $92(40.71)$ & $134(59.29)$ & $1.58(0.84,2.95)$ & $2.15(0.79,5.81)$ \\
Marital & $36-49$ & $17(30.36)$ & $39(69.64)$ & 1 & 1 \\
status & Unmarried. & $14(60.87)$ & $9(39.13)$ & $2.48(1.05,5.89)$ & $2.56(0.87,4.58)$ \\
Educational & Married & $131(38.53)$ & $209(61.47)$ & 1 & 1 \\
status & $\leq 4$ Grade & $12(48.00)$ & $13(52.00)$ & $3.08(0.92,10.25)$ & $1.07(0.24,4.75)$ \\
& $5-12$ Grade & $98(42.24)$ & $134(57.76)$ & $2.44(0.94,6.29)$ & $0.51(0.10,2.55)$ \\
Residence & $>12$ Grade & $6(23.08)$ & $20(76.92)$ & 1 & 1 \\
& Urban & $62(31.00)$ & $138(69.00)$ & 1 & 1 \\
& Rural & $83(50.92)$ & $80(49.08)$ & $2.31(1.50,3.55)$ & $1.89(0.98,3.68)$ \\
Family size & $\leq 5$ & $103(33.55)$ & $204(66.45)$ & 1 & 1 \\
& $>5$ & $42(75.00)$ & $14(25.00)$ & $5.94(3.10,11.38)$ & $7.74(4.15,16.47)$ \\
Parity & Primigravida & $11(31.43)$ & $24(68.57)$ & 1 & 1 \\
& Multigravida & $134(40.85)$ & $194(59.15)$ & $2.51(1.71,3.18)$ & $2.66(1.31,4.53)$ \\
Household & $\leq 968$ ETB & $97(52.15)$ & $89(47.85)$ & $6.12(3.26,8.62)$ & $5.81(2.93,14.11)$ \\
income* & $>968$ ETB & $48(27.12)$ & $129(72.88)$ & 1 & 1 \\
\hline
\end{tabular}

NB: COR = Crude Odds Ratio, AOR = Adjusted Odds Ratio, C.I = Confidence Interval, Household income $*$ Household income per month in Ethiopian birr 
Table 2: Association of anemia with clinical, reproductive health and nutrition related factors among pregnant women attending ANC clinic in Wolayita Town, Southern Ethiopia: January to March 2014

\begin{tabular}{|c|c|c|c|c|c|}
\hline \multirow[t]{2}{*}{ Variables } & \multirow[t]{2}{*}{ Categories } & \multicolumn{2}{|c|}{ Anemia } & \multirow[t]{2}{*}{ COR (95\% C.I) } & \multirow[t]{2}{*}{$\operatorname{AOR}(95 \%$ C.I $)$} \\
\hline & & Yes (\%) & No (\%) & & \\
\hline \multirow[t]{2}{*}{ Clinical illness } & Yes & 73 (67.59) & $35(32.41)$ & $5.30(3.26,8.62)$ & $6.38(3.13,13.00)$ \\
\hline & No & $72(28.24)$ & $183(71.76)$ & 1 & 1 \\
\hline \multirow{2}{*}{ History of malaria } & Yes & $22(52.38)$ & $20(47.62)$ & $1.77(0.59,3.39)$ & $2.06(0.76,5.62)$ \\
\hline & No & $123(38.31)$ & $198(61.69)$ & 1 & 1 \\
\hline \multirow[t]{2}{*}{ Malaria infection } & Yes & $7(53.85)$ & $6(46.15)$ & $1.79(0.59,5.45)$ & \\
\hline & No & $138(39.43)$ & $212(60.57)$ & 1 & \\
\hline \multirow[t]{2}{*}{ Presence of current IPI } & Yes & $40(57.97)$ & $29(42.03)$ & $2.48(1.46,4.24)$ & $2.41(1.08,5.81)$ \\
\hline & No & $105(35.71)$ & $189(64.29)$ & 1 & 1 \\
\hline \multirow{4}{*}{$\begin{array}{l}\text { History of } \\
\text { contraceptive usage } \\
\text { Excess menstrual } \\
\text { bleeding }\end{array}$} & Yes & $108(35.64)$ & $195(64.36)$ & 1 & 1 \\
\hline & No & 37 (61.67) & $23(38.33)$ & $2.91(1.64,5.14)$ & $5.02(2.21,11.47)$ \\
\hline & Yes & $29(76.32)$ & $9(23.68)$ & $5.81(2.66,12.86)$ & $9.82(3.27,21.35)$ \\
\hline & No & $116(35.69)$ & $209(64.31)$ & 1 & 1 \\
\hline \multirow[t]{3}{*}{ Trimesters } & $1^{\text {st }}$ & $12(22.64)$ & $41(77.36)$ & 1 & 1 \\
\hline & $2^{\text {nd }}$ & $69(33.17)$ & 139 (66.83) & $1.69(0.84,3.43)$ & $3.39(1.05,9.89)$ \\
\hline & $3^{\text {rd }}$ & $64(62.75)$ & $38(37.25)$ & $5.75(2.69,12.28)$ & $11.37(4.56,24.82)$ \\
\hline \multirow[t]{2}{*}{ History of abortion } & Yes & $18(43.90)$ & $23(56.10)$ & $1.20(0.62,2.32)$ & \\
\hline & No & $127(39.44)$ & $195(60.56)$ & 1 & 1 \\
\hline \multirow{3}{*}{$\begin{array}{l}\text { Body Mass Index in } \\
\mathrm{kg} / \mathrm{m}^{2}\end{array}$} & Low & $30(78.95)$ & $8(21.05)$ & $12.45(9.07,91.17)$ & $9.44(7.79,22.18)$ \\
\hline & Normal & 109 (39.93) & $164(60.07)$ & $5.09(2.10,12.34)$ & $5.72(3.05,7.70)$ \\
\hline & High & $6(11.5)$ & $46(88.5)$ & 1 & 1 \\
\hline
\end{tabular}

NB: $\mathrm{COR}=$ Crude Odds Ratio, C.I = Confidence Interval, AOR = Adjusted Odds Ratio, IPI = Intestinal Parasitic Infection

\section{DISCUSSION}

According to the WHO cutoff values (1), prevalence of anemia in this study indicated not much lower than sever public heath importance. This result was consistent with study done in Jimma University Specialized Hospital, South West Ethiopia, 2006 (38.2\%) (9) and West Arsi Zone, Ethiopia, 2013 (36.6\%) (7). However, the result of the present study was much lower than WHO report, 2008 (62.7\%) in Ethiopia (1). This might have happened due to the model used to estimate prevalence of anemia and the time difference.

The prevalence of anemia in this study is also low as compared to the prevalence of anemia reported from Morogoro municipality, Tanzania, 2010 (13), South Eastern Nigeria, 2007 (2), Sudan, 2009 (4), Kenya, 2007 (5), Uganda, 2013 (18), China, 2009 (19), Malaysia, 2012 (20) and Gilgel Gibe dam area, Southwest Ethiopia, 2012 (8) which reported 95\%, 76.9\%, 70\%, 69.1\%, $63.1 \%, 58.6 \%, 57.4 \%$ and $53.9 \%$ prevalence rates, respectively. This variation might be due to study population difference. For instance, in Tanzania where the highest prevalence was reported, most of the participants were in their last trimester whereas most of the participants in our study were in the $2^{\text {nd }}$ and $1^{\text {st }}$ trimester. The other possible reason might be due to low prevalence of malaria compared to other studies; for example, a study in Gilgel Gibe Dam area, Southwest Ethiopia, 2012 (8) reported $11.60 \%$ of malaria cases among pregnant women while the prevalence of malaria was $3.58 \%$ in our study.

On the other hand, prevalence of anemia in this study was higher than similar studies conducted in Nakhonsawan, Thailand 2010 (21), Azezo Health Center Gondar Town, North West Ethiopia 2013 (22), and Tikur Anbessa Specialized Hospital, Addis Ababa Ethiopia 2014 (6) which reported as $14.1 \%, 21.3 \%$ and $21.6 \%$, respectively. This might be due to a difference in socio-economic and educational status between the study populations. For example, the current study indicated that $6.89 \%$ and $7.61 \%$ of the study participants were illiterate and had educational status of greater than Grade 12, respectively, while 
similar study in Tikur Anbessa Specialized Hospital indicated $5.07 \%$ and $30.89 \%$, respectively. The other possible reason might be methodological variation as Sahli-Hellige method was used for the determination of $\mathrm{Hb}$ in a study in Northwest Ethiopia (22) which is prone to personal observation bias.

According to WHO classification for degree of anemia, among anemic pregnant women, 14 $(3.89 \%)$ were severely anemic. The proportion of severe anemia in this study was higher compared to similar studies in Nigeria 2014 (0.3\%) (23), Sudan 2010 (2.1\%) (4) and Tanzania 2011 (2.1\%) (24). Mainly, this might be due to the method used for measurement of $\mathrm{Hb}$ level of pregnant women. The study conducted in Nigeria used packed cell volume or hematocrit for identification of anemic cases (23), but in our case, $\mathrm{Hb}$ was measured using a HemoCue hemoglobinometer (HemoCue $\mathrm{AB}$, Angelhom, Sweden) $(4,24)$ which is accurate and precise method for measurement of $\mathrm{Hb}$. This method we used is a recommend method for field research work.

Anemia in pregnancy is related to different socio-demographic factors $(6,21,25)$. This study assessed socio-demographic variables associated with anemia. Age group of 15-24 years, family size greater than four, multigravida and monthly household income less than 968 Ethiopian Birr showed statistically significant association with anemia. This indicates a higher prevalence of anemia in young pregnant mothers, large family size and large number of children ever borne. In different studies, age, family size, parity and economical status were found to be significantly associated with anemia during pregnancy $(6,21$, $25)$ which were consistent with our study. Reports on prevalence of anemia in women of reproductive age in Meghalaya, 2010 (26) and in India, 2008 (27) were consistent with the current finding.

In this study, anemia among pregnant women was significantly associated with presence of clinical illness. Pregnant women who had clinical illness and intestinal parasitic infection were more likely to be anemic than pregnant women who did not have clinical illness and intestinal parasitic infections. Similar findings were obtained from Nigeria (2) and Ethiopia (8). In the present study, anemia was more prevalent at the third trimester. This might be due the fact that increase in trimester may cause reduction in maternal iron reserves. There was also a statistically significant association between anemia and history of excess menstrual bleeding (usage of more than two sanitary pads within a day during menstruation). These might be due to the fact that increase in number of sanitary pad usage per day reflects increase in the amount of blood flow-one of the predisposing factors for the occurrence of anemia. Undernourished pregnant women who had low BMI $<18.5 \mathrm{~kg} / \mathrm{m}^{2}$ were more likely to have anemia. This might be due to the fact that anemia is one of the most common nutritional deficiency disorders. Because the study was cross-sectional in design, it did not show which preceded, anemia or risk factors. Additionally, Micronutrient (serum iron, folate and vit- $\mathrm{B}_{12}$ ) levels, which might be root causes of anemia, were not assessed.

In conclusion, the overall prevalence of anemia in this study indicated that it is a moderate public health problem. Intervention strategies should focus on associated factors of anemia among pregnant women. Increase awareness for family planning methods might have a contribution for reducing risk of anemia. Economic and nutritional empowerment should be considered. Large scale longitudinal studies should be done to identify specific etiologies and root causes of anemia among pregnant women by assessing micronutrients (serum iron, folate and vit-B 12 levels).

\section{ACKNOWLEDGMENTS}

We would like to thank staff members of Wolayita Soddo Otona Hospital, particularly the Medical laboratory staff for their cooperation during data collection. We thank Jimma University for financial support. We are also grateful to the study participants.

\section{REFERENCES}

1. Benoist B, McLean E, Cogswell M, Egli I, Wojdyla D. Worldwide prevalence of anemia, WHO Vitamin and Mineral Nutrition Information System, 1993-2005. Public Health Nutr, Geneva 2008; 12(4): 444-454.

2. Uneke CJ, Duhlinska DD, Igbinedion EB. Prevalence and public-health significance of HIV infection and anemia among pregnant women attending antenatal clinics in south- 
eastern Nigeria. J Health Popul Nutr, 2007; 25(3):328-335.

3. Antelman G, Msamanga GI, Spiegelman D, et al. Nutritional factors and infectious disease contribute to anemia among pregnant women with human immunodeficiency virus in Tanzania. J Nutr, 2000; 130(8):1950-7.

4. Haggaz AD, Radi EA, Adam I. Anemia and low birth weight in western Sudan. Trans $R$ Soc Trop Med Hyg, 2010; 104(3):234-6.

5. Ouma P, van Eijk AM, Hamel MJ, et al. Malaria and anemia among pregnant women at first antenatal clinic visit in Kisumu, western Kenya. Trop Med Int Health, 2007; 12(12):1515-23.

6. Jufar AH, Zewde T. Prevalence of Anemia among Pregnant Women Attending Antenatal Care at TikurAnbessa Specialized Hospital, Addis Ababa Ethiopia. J Hematol Thrombo, Dis2013; 2(1): 1-6.

7. Obse N, Mossie A, Gobena T. Magnitude of Anemia and Associated Risk Factors among Pregnant Women Attending Antenatal Care in Shalla Woreda, West Arsi Zone, Oromia Region, Ethiopia. Ethiop J Health Sci, 2013; 23(2):165-73.

8. Getachew M, Yewhalaw D, Tafess K, Getachew Y, Zeynudin A. Anemia and associated risk factors among pregnant women in Gilgel Gibe dam area, Southwest Ethiopia. Parasites \& amp; vectors, 2012; 5(1):296.

9. Belachew T, Legesse Y. Risk factors for anemia among pregnant women attending antenatal clinic at Jimma University Hospital, southwest Ethiopia. Ethiop Med J, 2006; 44(3):211-20.

10. Allen LH. Anemia and iron deficiency: effects on pregnancy outcome. Am J Clin Nutr 2000; 71(5):1280S-4S.

11. Lone FW, Qureshi RN, Emmanuel F. Maternal anemia and its impact on perinatal outcome in a tertiary care hospital in Pakistan. East Mediter Health J, 2004; 10(6):801-7.

12. Melku M, Addis Z, Alem M, Enawgaw B. Prevalence and Predictors of Maternal Anemia during Pregnancy in Gondar, Northwest Ethiopia: An Institutional Based CrossSectional Study. Anemia, 2014; 2014: 108593.

13. Msolla MJ, Kinabo JL. Prevalence of anemia in pregnant women during the last trimester. Int J Food Sci Nutr, 1997; 48(4):265-70.
14. Kidanto HL, Mogren I, Lindmark G, Massawe S, Nystrom L. Risks for preterm delivery and low birth weight are independently increased by severity of maternal anemia. S Afr Med J, 2009; 99(2):98-102.

15. Iron deficiency anemia: assessment, prevention, and control. A guide for programme managers. Geneva, WHO 2001.

16. Tolentino K, Friedman JF. An update on Anemia in Less Developed Countries. Am J Trop Med Hygiene, 2007; 77(1): 44-51.

17. WHO. Hemoglobin Concentrations for the Diagnosis of Anemia and Assessment of Severity, Vitamin and Mineral Nutrition Information System, WHO, Geneva, Switzerland. 2011.

18. Mbule MA, Byaruhanga YB, Kabahenda M, Lubowa A. Determinants of anemia among pregnant women in rural Uganda. Rural Remote Health, 2013; 13(2):2259.

19. Ma AG, Schouten E, Wang Y, et al. Anemia prevalence among pregnant women and birth weight in five areas in China. Med Princ Pract, 2009; 18(5): 368-72.

20. Rosmawati NK, Nazri SM, Ismail IM. The Rate and Risk Factors for Anemia among Pregnant Mothers in Jerteh Terengganu, Malaysia. J Community Med Health Educ, 2012; 2(5).

21. Sukrat B, Suwathanapisate P, Siritawee S, Poungthong T, Phupongpankul K. The prevalence of iron deficiency anemia in pregnant women in Nakhonsawan, Thailand. $J$ Med Assoc Thai, 2010; 93 (7):765-70.

22. Meseret A, Bamlaku E, Aschalew G, Tigist K, Mohamed S, Yadesa O. Prevalence of Anemia and Associated Risk Factors among Pregnant Women Attending Antenatal Care in Azezo Health Center Gondar Town, Northwest Ethiopia. J Int Histo, 2013; 1(3): 137-44.

23. Olatunbosun OA, Abasiattai AM, Bassey EA, James RS, Ibanga G, Morgan A. Prevalence of Anemia among Pregnant Women at Booking in the University of Uyo Teaching Hospital, Uyo, Nigeria. Biomed Res Int, 2014; 2014: Article ID: 849080.

24. Msuya SE, Hussein TH, Uriyo J, Sam NE, Stray-Pedersen B. Anemia among pregnant women in northern Tanzania: prevalence, risk factors and effect on perinatal outcomes. Tanzan J Health Re, 2011; 13(1): 33-9. 
25. Karaoglu L, Pehlivan E, Egri M, et al. The prevalence of nutritional anemia in pregnancy in an east Anatolian province, Turkey. $B M C$ Public Health, 2010; 10: 329.

26. Sanku D, Sankar G, Madhuchhanda G. Prevalence of anemia in women of reproductive age in Meghalaya: a logistic regression analysis. Turk J Med Sci, 2010; 40 (5):783-789.

27. Bharati P, Som S, Chakrabarty S, Bharati S, Pal M. Prevalence of anemia and its determinants among non-pregnant and pregnant women in India. Asia Pac J Public Health, 2008; 20(4):347-59. 